\title{
Infectious Causes of Eosinophilic Meningitis in Korean Patients: A Single-Institution Retrospective Chart Review from 2004 to 2018
}

\author{
Sunghee Park, Jiwon Jung, Yong Pil Chong, Sung-Han Kim, Sang-Oh Lee, Sang-Ho Choi, Yang Soo Kim, \\ Min Jae Kim* (i)
}

Division of Infectious Diseases, Department of Internal Medicine, Asan Medical Center, University of Ulsan College of Medicine, Seoul 05505, Korea

\begin{abstract}
Eosinophilic meningitis is defined as the presence of more than 10 eosinophils per $\mu$ in the cerebrospinal fluid (CSF), or eosinophils accounting for more than $10 \%$ of CSF leukocytes in patients with acute meningitis. Parasites are the most common cause of eosinophilic meningitis worldwide, but there is limited research on patients in Korea. Patients diagnosed with eosinophilic meningitis between January 2004 and June 2018 at a tertiary hospital in Seoul, Korea were retrospectively reviewed. The etiology and clinical characteristics of each patient were identified. Of the 22 patients included in the study, 11 (50\%) had parasitic causes, of whom 8 (36\%) were diagnosed as neurocysticercosis and 3 (14\%) as Toxocara meningitis. Four (18\%) patients were diagnosed with fungal meningitis, and underlying immunodeficiency was found in 2 of these patients. The etiology of another 4 (18\%) patients was suspected to be tuberculosis, which is endemic in Korea. Viral and bacterial meningitis were relatively rare causes of eosinophilic meningitis, accounting for 2 (9\%) and 1 (5\%) patients, respectively. One patient with neurocysticercosis and 1 patient with fungal meningitis died, and 5 (23\%) had neurologic sequelae. Parasite infections, especially neurocysticercosis and toxocariasis, were the most common cause of eosinophilic meningitis in Korean patients. Fungal meningitis, while relatively rare, is often aggressive and must be considered when searching for the cause of eosinophilic meningitis.
\end{abstract}

Key words: Eosinophilic meningitis, etiology, Korea

\section{INTRODUCTION}

Eosinophilic meningitis is a rare clinical entity defined as eosinophilic pleocytosis in the cerebrospinal fluid (CSF) of patients with symptoms suggestive of meningitis. Parasites such as Angiostrongylus cantonensis and Gnathostoma spinigerum are common causes of eosinophilic meningitis in regions such as North America, Southeast Asia, and the Caribbean islands [1-4]. However, there has been limited research on the causes and characteristics of eosinophilic meningitis in Korea. While there have been occasional case reports of eosinophilic meningitis due to atypical pathogens $[5,6]$, only 1 study describes the etiology and characteristics of eosinophilic meningitis in Korean children [7]. However, $90 \%$ of the patients included in this study were post-neurosurgical patients, and thus there were

\footnotetext{
- Received 26 March 2021, Revised 3 June 2021, Accepted 3 June 2021.

* Corresponding author (nahani99@gmail.com)

(C) 2021, Korean Society for Parasitology and Tropical Medicine

This is an Open Access article distributed under the terms of the Creative Commons

Attribution Non-Commercial License (https://creativecommons.org/licenses/by-nc/4.0) which permits unrestricted non-commercial use, distribution, and reproduction in any

medium, provided the original work is properly cited.
}

limitations in identifying the microbiological causes of eosinophilic meningitis. In this study, we aimed to identify the common pathogens of eosinophilic meningitis and compare the characteristics of the patients according to their etiology.

\section{MATERIALS AND METHODS}

\section{Ethics statement}

The Institutional Review Board of Asan Medical Center reviewed and approved this study (\#2020-1060). The need for informed consent was waived by the committee considering the retrospective nature of the study.

\section{Study population}

We performed a retrospective review of medical records to identify patients with eosinophilic meningitis. We first identified patients who received lumbar punctures between January 2004 and June 2018 at a 2,700-bed tertiary referral hospital in Seoul, Korea. Among them, patients who met the following criteria were selected: (1) more than 10 eosinophils per $\mu$ in the CSF or eosinophils accounting for more than 10 percent of 
leukocytes in the CSF; and (2) clinical symptoms and signs suggestive of acute meningitis (e.g., headache, newly developed focal neurologic deficits, altered mental status, fever, or neck stiffness). Those who were diagnosed with non-infectious diseases such as demyelinating disease, malignancy and postoperative conditions were excluded. We collected demographic, clinical, laboratory, and radiologic data on the patients included in the final analysis. Peripheral blood eosinophilia was noted if the complete blood cell count revealed eosinophils > 500 per $\mu$ l or more than $10 \%$ of the white blood cell count.

\section{Criteria for classification of etiology}

The etiology was considered to be confirmed when CSF or blood culture, CSF virus polymerase chain reaction (PCR), or CSF cryptococcal antigen tests had positive results for the causative microorganism. Meningitis associated with neurocysticercosis was considered when a patient had computed tomography (CT) or magnetic resonance imaging (MRI) findings suggestive of neurocysticercosis (e.g., intraparenchymal, intraventricular, or subarachnoid cystic lesions, calcifications, leptomeningeal enhancement, or hydrocephalus with ventricular enlargement) and positive anti-cysticercus antibodies in the CSF [3]. The results of serum anti-cysticercus antibodies were not used considering its relatively poor sensitivity and specificity [8]. Meningitis associated with toxocariasis was considered when a patient had symptoms such as headache, seizure, focal neurologic deficits, confusion, or cognitive impairment [9], along with a history of raw meat intake and positive results for serum anti-Toxocara antibodies. Patients with suspected tuberculous (TB) meningitis were classified as definite, probable, or possible according to consensus guidelines outlined in a previous study [10]. Only those diagnosed with definite or probable TB meningitis were included in the TB meningitis group.

\section{RESULTS}

During the study period, 739 events of CSF eosinophilic pleocytosis were identified in 314 patients. Two hundred and thirty patients had secondary eosinophilic pleocytosis due to other medical conditions including postoperative status, ventriculoperitoneal shunt infections, and leptomeningeal metastasis of malignancy. Of the remaining 84 patients, 62 were clinically diagnosed as having either non-infectious conditions such as transverse myelitis and multiple sclerosis, or meningoencephalitis of unknown etiology. Twenty-two patients had probable or confirmed infectious etiologies and were thus included in the final analysis. Their etiologies were as seen in Table 1: neurocysticercosis $(n=8,36 \%)$, fungal meningitis $(n=4$, $18 \%)$, TB meningitis $(\mathrm{n}=4,18 \%)$, Toxocara meningitis $(\mathrm{n}=3$, $14 \%)$, viral meningitis $(\mathrm{n}=2,9 \%)$, and bacterial meningitis $(\mathrm{n}=1,5 \%)$.

Neurocysticercosis was the most common etiology among the patients included in our study. Six (75\%) patients were male, and the mean age of the neurocysticercosis group $(59.9 \pm 11.2$ years) was higher than those of other groups. Gait disturbance was reported in half of the patients. Hydrocephalus and meningeal enhancement on brain imaging were found in $7(88 \%)$ and $2(25 \%)$ patients, while steroids, albendazole, and praziquantel were prescribed in $5(63 \%), 4(50 \%)$, and 3 (38\%) patients, respectively. One patient died due to the disease and 5 patients $(63 \%)$ had neurologic sequelae, of which gait disturbance or lower extremity weakness were the most common symptoms.

One of the patients was a 66 year old male with no previous medical history, who was admitted with symptoms of headache, vertigo, and gait disturbance. He had a history of working in Libya 35 years ago, and a history of travel to Vietnam (4 years ago) and China (1 year ago). CSF findings showed eo-

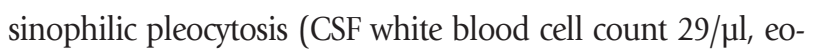
sinophil percentage $18 \%$ ), with elevated protein levels (89.3 $\mathrm{mg} / \mathrm{dl}$ ) and normal glucose levels $(56 \mathrm{mg} / \mathrm{dl}$ ). Brain MRI showed multiple cystic lesions with focal wall enhancement at both frontal base and subcallosal areas, with ventriculomegaly of both lateral ventricles and the 3rd ventricle (Fig. 1). Multiple calcific nodules in soft tissue were seen on chest imaging (Fig. 2). Based on these findings, immunodiagnostic testing for parasite infections were performed on the patient's blood and CSF. Both serum and CSF cysticercus antibodies were positive, and the patient was diagnosed with neurocysticercosis. Albendazole was administered for 4 weeks, in combination with dexamethasone. Afterwards, his symptoms of headache improved, but his limping gait worsened, leading to a second course of therapy with albendazole, praziquantel, and steroids 3 months after the initial treatment. The patient showed slight improvement in symptoms before he was transferred to another hospital, and follow-up was lost.

All of the patients in the Toxocara meningitis group were male and had a history of raw meat intake. All 3 patients reported headache, while vomiting and neck stiffness were also seen in 1 patient. MRI findings suggestive of ventriculitis with 


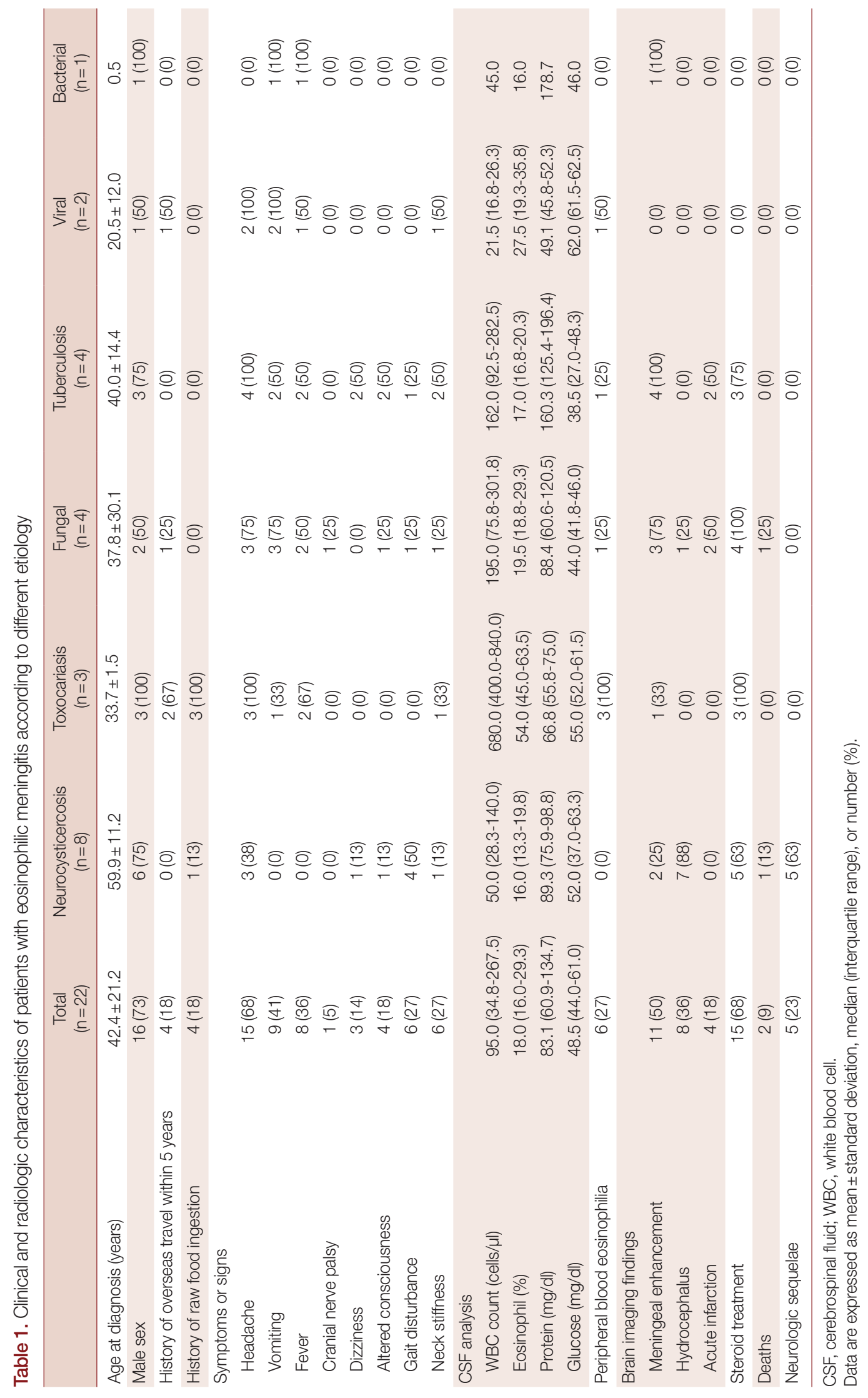



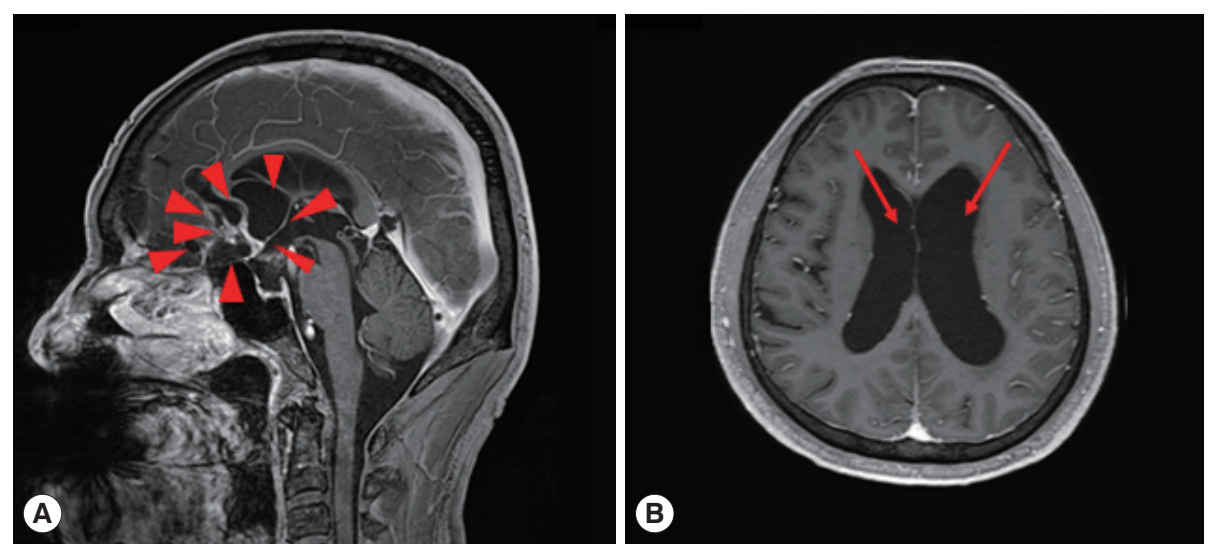

Fig. 1. Brain magnetic resonance imaging (MRI) of a patient diagnosed with neurocysticercosis. (A) Multiple cystic lesions with focal wall enhancement are seen at both frontal base and subcallosal areas, suggestive of parasitic cysts (red arrowheads). (B) Ventriculomegaly of both lateral ventricles (red arrows) can be seen.

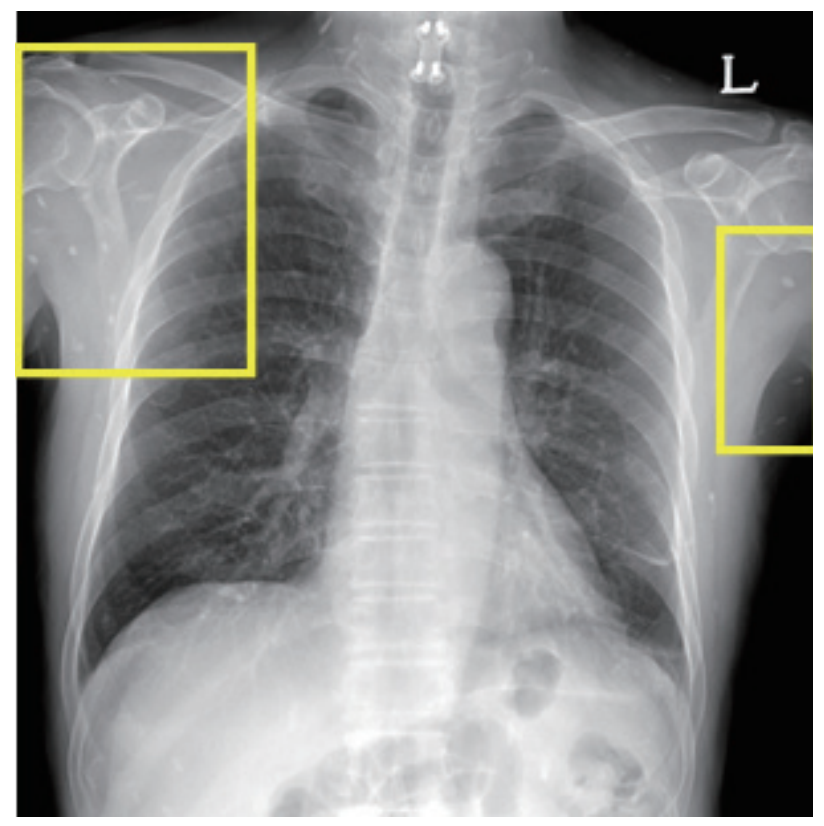

Fig. 2. Chest radiograph of a neurocysticercosis patient. Numerous small calcific lesions in the soft tissue can be seen, suggestive of calcified granulomas related to cysticercosis (easily found in the yellow rectangular areas).

choroid plexitis in the right lateral ventricle were seen in one patient, while the others had normal brain MRIs. Abdominopelvic CT in one patient showed benign inflammatory lesions suspicious of eosinophilic infiltrations in the liver. The median CSF WBC count was 680 cells $/ \mu \mathrm{l}$, and the median eosinophil percentage was $54 \%$. All of the patients had peripheral blood eosinophilia. Two patients were treated with albendazole and steroids, while only steroids were used in 1 patient. There were no deaths or neurologic sequelae in this group.
In the fungal meningitis group, Cryptococcus neoformans and Exophiala dermatitidis were found to be the causative pathogens, while in 1 patient, the eosinophilic meningitis was associated with rhinocerebral mucormycosis. Prototheca wickerhamii, an alga that is ubiquitously found in nature, was repeatedly found in the CSF culture of another patient, and the patient was included in the fungal group. The patient with recurrent Prototheca wickerhamii meningitis and the patient infected with Exophiala dermatitidis were both children under the age of 15. While both children were healthy before the episode of meningitis, they were both later diagnosed with Caspase Recruitment Domain 9 (CARD9) deficiency. The other 2 patients were adults and did not have a history of immunodeficiency. All patients with fungal meningitis were treated with appropriate antifungal agents, and the patient with Exophiala dermatitidis meningitis died due to the disease. Of the surviving patients, none had neurologic sequelae.

Among the patients with TB meningitis, all showed meningeal enhancement on brain imaging by CT or MRI, and 2 (50\%) had acute infarctions. All 4 patients were treated with anti-tuberculosis drugs and 3 patients were treated with adjunctive steroids. There were no deaths or neurologic sequelae in this group.

Two patients were diagnosed with viral meningitis, with positive CSF PCR results for enterovirus and herpes simplex virus-2, respectively. There were no abnormalities on brain imaging, and both patients survived without any neurologic sequelae. Enterobacter aerogenes was cultured in the CSF of a 5-month-old patient with a history of recurrent meningitis and intraabdominal surgery due to a congenital anomaly. The 
patient survived after receiving appropriate antibiotic therapy.

\section{DISCUSSION}

In this retrospective study, we examined the main causative microorganisms of eosinophilic meningitis in Korean patients. In contrast to previous studies that were mostly case series reporting atypical etiologies $[5,6]$, our study systematically reviewed the etiology of community-acquired eosinophilic meningitis. Neurocysticercosis was the most common cause, comprising $36 \%$ of the cases, followed by fungal, $\mathrm{TB}$, and Toxocara meningitis, while bacteria and viruses accounted for less than $15 \%$ of the etiology. Among the 4 patients who had fungal eosinophilic meningitis, 2 were children who were later identified as having an underlying immune deficiency condition. One patient with neurocysticercosis and 1 patient with fungal meningitis died, while $23 \%$ of patients reported having neurologic sequelae.

Neurocysticercosis was the most common etiology of eosinophilic meningitis in our study. Patients with neurocysticercosis were older than those with other etiologies, which likely reflects the long incubation period of the disease and its decreasing incidence in Korea. All 8 patients with neurocysticercosis had extraparenchymal involvement, such as subarachnoid or ventricular lesions, while 4 also had parenchymal lesions. The extraparenchymal involvement could have been associated with the high prevalence of hydrocephalus and related symptoms of gait disturbance in this group. It is also consistent with the results of a previous study that showed extraparenchymal neurocysticercosis to be associated with inflammatory changes in the CSF [11]. Several patients had recurrent bouts of meningitis due to neurocysticercosis. These results suggest that cysticercus antibody screening may be considered in elderly patients with eosinophilic meningitis.

Another notable parasitic cause of eosinophilic meningitis was toxocariasis. Several case reports of eosinophilic meningoencephalitis caused by toxocariasis have been published, and the authors of a literature review have suggested that Toxocara species infection should be considered in the differential diagnosis of eosinophilic meningitis [12]. Patients in the toxocariasis group had the highest median CSF WBC count and eosinophil percentage, and all of them had peripheral blood eosinophilia. One thing to note is that all 3 patients had a history of raw meat ingestion. Toxocariasis usually occurs through the ingestion of Toxocara species eggs, but raw meat consumption is also considered to be a potential source [13-15]. In Korea, the consumption of a cow's raw meat or raw liver is still popular and considered to be a cause of various parasitic diseases, including toxocariasis. Since toxocariasis usually has a benign clinical course, it is possible that many cases of Toxocara meningitis go unnoticed and undiagnosed. Also, as can be seen from the patient in this study who improved after receiving steroids alone, basing the diagnosis of toxocariasis on the patient's response to anthelminthic treatment is also unreliable. The treatment of toxocariasis is more focused on the control of inflammation by using steroids rather than killing the parasites by anthelminthics [16]. The larva of Toxocara species do not undergo any further development in human tissues. Instead, the human inflammatory response can cause damage to tissues, resulting in clinical toxocariasis. It is also possible that Toxocara was incorrectly considered as a causative agent in some cases since serum anti-Toxocara antibodies are notorious for their longevity $[17,18]$, and testing for anti-Toxocara antibodies in the CSF was not performed in this study. Thus, further research is urgently needed to identify whether Toxocara could be the real pathogenic microorganism in patients with a history of raw meat intake, eosinophilic pleocytosis in the CSF, and peripheral blood eosinophilia, and CSF testing for antiToxocara antibodies should be actively performed in patients meeting the previous criteria.

Fungal meningitis was diagnosed in approximately $1 / 5$ of the patients with eosinophilic meningitis. Fungal meningitis has mostly been reported in immunocompromised individuals with neutropenia or those who have received surgery, chemotherapy, or central catheterization [19]. In children and neonates, prior neurosurgery, cancer, ventriculoperitoneal shunts, and previous broad-spectrum antibiotic use were reported to be risk factors [20]. Among the 4 patients diagnosed with fungal eosinophilic meningitis in our study, 2 were children under the age of 15 who did not have risk factors for fungal infection at the time of diagnosis. Both children were later diagnosed with CARD9 deficiency, an inherited condition associated with life-threatening invasive fungal infections due to impairments in cytokine production, neutrophil recruitment, and Th17 immunity [21]. Invasive fungal infections have a high rate of mortality unless promptly treated; therefore, it should be noted that fungal eosinophilic meningitis could be the presenting infection of an inherited immune deficiency disorder in previously healthy children.

TB is a well-known endemic disease in Korea, and TB men- 
ingitis is a common cause of meningoencephalitis in this country. In a study performed at a tertiary hospital in Korea in 1997 on 241 patients diagnosed with infectious meningitis, the incidence rate of TB meningitis was approximately $24 \%$ [22]. The high incidence rate of TB meningitis in Korea was most likely reflected in the proportion of TB among the patients included in this study. Considering that delayed treatment of TB meningitis is often associated with poor prognosis, TB meningitis should be considered as a possible etiology in patients with eosinophilic meningitis.

Since the study population was relatively small, future studies with a bigger population may reveal more diverse causes and different distribution patterns. Also, because this was a retrospective study, the diagnostic tests performed on the patients were not standardized. It is possible that the causative pathogens of some of the patients with unknown etiology might have been determined if comprehensive testing had been uniformly carried out. The incidence of TB and toxocariasis were notably higher than those reported in the literature, most likely due to their endemic status in Korea, and caution is needed when applying the results of the current study in countries with different epidemiological risk factors. Lastly, there were limitations in the precise diagnosis of TB meningitis and toxocariasis. TB meningitis was diagnosed based on clinical criteria with or without microbiological confirmation, while the diagnosis of toxocariasis was based on patient history and positive serum anti-Toxocara antibodies, which are known for their prolonged persistence.

In conclusion, among Korean patients with eosinophilic meningitis, parasitic diseases such as neurocysticercosis and toxocariasis were the most common cause. The proportion of fungal meningitis was relatively low, but it should not be ignored considering its high mortality rate. $\mathrm{TB}$, viral, and bacterial causes were uncommon as well, but they should not be overlooked when searching for the cause in patients with eosinophilic meningitis. Further prospective studies with larger study populations are warranted for more widely applicable results.

\section{ACKNOWLEDGMENTS}

This research did not receive any specific grant from funding agencies in the public, commercial, or not-for-profit sectors.

\section{CONFLICT OF INTEREST}

The authors declare no conflict of interest regarding this study.

\section{REFERENCES}

1. Weller PF, Liu LX. Eosinophilic meningitis. Semin Neurol 1993; 13: 161-168. https://doi.org/10.1055/s-2008-1041121

2. Ramirez-Avila L, Slome S, Schuster FL, Gavali S, Schantz PM, Sejvar J, Glaser CA . Eosinophilic meningitis due to Angiostrongylus and Gnathostoma species. Clin Infect Dis 2009; 48: 322-327. https://doi.org/10.1086/595852

3. Graeff-Teixeira C, da Silva AC, Yoshimura K. Update on eosinophilic meningoencephalitis and its clinical relevance. Clin Microbiol Rev 2009; 22: 322-348, Table of Contents. https://doi. org/10.1128/cmr.00044-08

4. Lo Re V 3rd, Gluckman SJ. Eosinophilic meningitis. Am J Med 2003; 114: 217-223. https://doi.org/10.1016/s0002-9343(02)01495-x

5. Yu G, Kim DH, Lee SH, Hwang JW, Lim SK, Kim CB, Lee NH, Sim WE, Yang YM, Lee CI. Ten cases of eosinophilic meningitis. Korean J Med 1981; 24: 848-855 (in Korean). https://www.ekjm. org/journal/view.php?number=2691

6. Choi HY, Park HJ, Won YS, Jung JW, Park SC. A Case of Eosinophilic Meningitis Associated with Idiopathic Hypereosinophilic Syndrome. J Korean Neurol Assoc 2005; 23: 396-398 (in Korean). https://www.jkna.org/journal/view.php?number=5245

7. Byun JH, Choi SY, Kim DS, Kim KH. Clinical manifestation of eosinophilic meningitis in korean children: a single institution's experience. Pediatric Infection and Vaccine 2015; 22: $23-28$ (in Korean). https://doi.org/10.14776/piv.2015.22.1.23

8. White AC Jr, Coyle CM, Rajshekhar V, Singh G, Hauser WA, Mohanty A, Garcia HH, Nash TE. Diagnosis and treatment of neurocysticercosis: 2017 Clinical practice guidelines by the Infectious Diseases Society of America (IDSA) and the American Society of Tropical Medicine and Hygiene (ASTMH). Clin Infect Dis 2018; 66: 49-75. https://doi.org/10.1093/cid/cix1084

9. Sánchez SS, García HH, Nicoletti A. Clinical and magnetic resonance imaging findings of neurotoxocariasis. Front Neurol 2018; 9: 53. https://doi.org/10.3389/fneur.2018.00053

10. Marais S, Thwaites G, Schoeman JF, Török ME, Misra UK, Prasad K, Donald PR, Wilkinson RJ, Marais BJ. Tuberculous meningitis: a uniform case definition for use in clinical research. Lancet Infect Dis 2010; 10: 803-812. https://doi.org/10.1016/s1473-3099(10)70138-9

11. Son HJ, Kim MJ, Jung KH, Choi S, Jung J, Chong YP, Kim SH, Lee SO, Choi SH, Kim YS, Woo JH, Jung BK, Song H, Chai JY. Neurocysticercosis: Clinical characteristics and changes from 26 years of experience in an university hospital in Korea. Korean J Parasitol 2019; 57: 265-271. https://doi.org/10.3347/kjp.2019.57.3.265

12. Vidal JE, Sztajnbok J, Seguro AC. Eosinophilic meningoencephalitis due to Toxocara canis: case report and review of the literature. 
Am J Trop Med Hyg 2003; 69: 341-343. https://pubmed.ncbi. nlm.nih.gov/14628955/

13. Karaca I, Menteş J, Nalçacı S. Toxocara neuroretinitis associated with raw meat consumption. Turk J Ophthalmol 2018; 48: 258261. https://doi.org/10.4274/tjo.27085

14. Choi D, Lim JH, Choi DC, Lee KS, Paik SW, Kim SH, Choi YH, Huh S. Transmission of Toxocara canis via ingestion of raw cow liver: a cross-sectional study in healthy adults. Korean J Parasitol 2012; 50: 23-27. https://doi.org/10.3347/kjp.2012.50.1.23

15. Choi D, Lim JH, Choi DC, Paik SW, Kim SH, Huh S. Toxocariasis and ingestion of raw cow liver in patients with eosinophilia. Korean J Parasitol 2008; 46: 139-143. https://doi.org/10.3347/ kjp.2008.46.3.139

16. Bennett J, Dolin R, Blaser MJ. Mandell, Douglas, and Bennett's Principles and Practice of Infectious Diseases. 9th ed. Philadelphia, USA. Elsevier. 2020, pp 3474.

17. Wilkins PP. Immunodiagnosis of human toxocariasis and prospects for improved diagnostics. Curr Trop Med Rep 2014; 1: 44-
51. https://doi.org/10.1007/s40475-013-0001-8

18. Fillaux J, Magnaval JF. Laboratory diagnosis of human toxocariasis. Vet Parasitol 2013; 193: 327-336. https://doi.org/10.1016/ j.vetpar.2012.12.028

19. Trupl J, Minarik T, Spanik S, Sufliarsky J, Krcmery V Jr. Nosocomial bacterial and fungal meningitis in cancer patients. Support Care Cancer 1995; 3: 425-427. https://doi.org/10.1007/bf00364985

20. Karabinis A, Hill C, Leclercq B, Tancrède C, Baume D, Andremont A. Risk factors for candidemia in cancer patients: a casecontrol study. J Clin Microbiol 1988; 26: 429-432. https://doi. org/10.1128/jcm.26.3.429-432.1988

21. Corvilain E, Casanova JL, Puel A. Inherited CARD9 Deficiency: Invasive Disease Caused by Ascomycete Fungi in Previously Healthy Children and Adults. J Clin Immunol 2018; 38: 656693. https://doi.org/10.1007/s10875-018-0539-2

22. Park SA, Cheon HY, Choi IS. Clinical Characteristics of Meningitis in Adults. J Korean Neurol Assoc 1997; 15: 1050-1063. https:// www.jkna.org/journal/view.php?year=1997\&vol=15\&spage=1050 
